\title{
BMJ Open The National Early Warning Score (NEWS) for outcome prediction in emergency department patients with community-acquired pneumonia: results from a 6-year prospective cohort study
}

\author{
Diana Sbiti-Rohr, ${ }^{1}$ Alexander Kutz, ${ }^{1}$ Mirjam Christ-Crain, ${ }^{2}$ Robert Thomann, ${ }^{3}$ \\ Werner Zimmerli, ${ }^{4}$ Claus Hoess, ${ }^{5}$ Christoph Henzen, ${ }^{6}$ Beat Mueller, ${ }^{1}$ \\ Philipp Schuetz, ${ }^{1}$ for the ProHOSP Study Group
}

To cite: Sbiti-Rohr D, Kutz A, Christ-Crain M, et al. The National Early Warning Score (NEWS) for outcome prediction in emergency department patients with community-acquired pneumonia: results from a 6 year prospective cohort study. BMJ Open 2016;6: e011021. doi:10.1136/ bmjopen-2015-011021

- Prepublication history for this paper is available online. To view these files please visit the journal online (http://dx.doi.org/10.1136/ bmjopen-2015-011021).

Received 4 January 2016 Revised 28 June 2016 Accepted 4 August 2016

CrossMark

For numbered affiliations see end of article.

Correspondence to Dr Philipp Schuetz; schuetzph@gmail.com

\section{ABSTRACT}

Objective: To investigate the accuracy of the National Early Warning Score (NEWS) to predict mortality and adverse clinical outcomes for patients with communityacquired pneumonia (CAP) compared to standard risk scores such as the pneumonia severity index (PSI) and CURB-65.

Design: Secondary analysis of patients included in a previous randomised-controlled trial with a median follow-up of 6.1 years.

Settings: Patients with CAP included on admission to the emergency departments (ED) of 6 tertiary care hospitals in Switzerland.

Participants: A total of 925 patients with confirmed CAP were included. NEWS, PSI and CURB-65 scores were calculated on admission to the ED based on admission data.

Main outcome measure: Our primary outcome was all-cause mortality within 6 years of follow-up. Secondary outcomes were adverse clinical outcome defined as intensive care unit (ICU) admission, empyema and unplanned hospital readmission all occurring within 30 days after admission. We used regression models to study associations of baseline risk scores and outcomes with the area under the receiver operating curve (AUC) as a measure of discrimination.

Results: 6 -year overall mortality was $45.1 \% \quad(n=417)$ with a stepwise increase with higher NEWS categories. For 30 day and 6 -year mortality prediction, NEWS showed only low discrimination (AUC 0.65 and 0.60 ) inferior compared to PSI and CURB-65. For prediction of ICU admission, NEWS showed moderate discrimination (AUC 0.73) and improved the prognostic accuracy of a regression model, including PSI (AUC from 0.66 to $0.74, p=0.001$ ) and CURB-65 (AUC from 0.64 to $0.73, p=0.015$ ). NEWS was also superior to PSI and CURB-65 for prediction of empyema, but did not well predict rehospitalisation.

Conclusions: NEWS provides additional prognostic information with regard to risk of ICU admission and

\section{Strengths and limitations of this study}

- This is the first large-scale study with a longterm follow-up investigating the association of the National Early Warning Score (NEWS) and adverse outcome in patients with community-acquired pneumonia.

- In the emergency department setting, NEWS was an adequate tool for risk stratification with regard to ICU admission and clinical empyema.

- The study was observational and it remains unclear whether NEWS will improve patient management.

- This study was limited to Swiss patients, predominantly Caucasian, limiting the generalisability of the results.

complications and thereby improves traditional clinicalrisk scores in the management of patients with CAP in the ED setting.

Trial registration number: ISRCTN95122877;

Post-results.

\section{INTRODUCTION}

Current guidelines recommend that clinical decisions regarding patient management in the emergency department (ED) setting are supported by objective risk scores. ${ }^{1-3}$ In patients with community-acquired pneumonia (CAP), risk scores support practitioners to decide whether a patient is at higher risk for mortality and, thus, may need inpatient treatment. ${ }^{4-7}$ Several risk scores have been developed and validated for predicting 30-day mortality in patients with $\mathrm{CAP}^{8-12}$ To date, the pneumonia severity index (PSI) and CURB-65 are recommended by most international guidelines for this purpose. ${ }^{2} 13$ 
CURB-65 is a five-point score that is predominantly used in Europe. The PSI is mostly used in the USA and has been validated in several studies. ${ }^{9}{ }^{14-19}$ As a limitation, both scores have their main focus on 30-day mortality prediction, but other outcomes such as disease severity (eg, requiring intensive care unit (ICU) admission) are not well predicted. ${ }^{20}$ This raises the question whether these scores can be improved by combination with other instruments focusing on the initial severity of disease, such as generalised early warning scores (EWS).

Among different EWS, the National Early Warning Score (NEWS), which was derived in the UK by the National Early Warning Score Development and Implementation Group (NEWSDIG) on behalf of the Royal College of Physicians, has been well established. ${ }^{21}$ Its purpose was to introduce a standardised trigger system to identify acutely ill patients throughout hospitalisation. NEWS consists of six physiological measurements classifying the patients into three risk categories (low, moderate, high). Several studies found NEWS to be superior compared to other risk stratification tools ${ }^{22-25}$ and a valid tool in different settings (ED, prehospital setting). ${ }^{26-28}$ Yet, there is currently no study investigating how well NEWS predicts severity and adverse clinical outcome in patients with CAP on admission to the ED.

Our hypothesis was that NEWS would show an association with short- and long-term adverse outcomes in patients with CAP and possibly improve risk prediction as compared to established CAP scores. The aim of our study was thus to compare the accuracy of NEWS with PSI and CURB-65 to predict mortality and adverse clinical outcomes in a well-characterised cohort of CAP patients from a previous randomised-controlled trial.

\section{METHODS}

\section{Study design}

This is a secondary analysis using data of 925 patients included in a previous randomised-controlled noninferiority trial with a 6 -year follow-up. The initial trial enrolled patients from October 2006 to March 2008 at six Swiss secondary or tertiary care, academic or nonacademic hospitals. ${ }^{29}$ The aim of the initial trial was to examine whether procalcitonin could reduce antibiotic use without compromising the safety of patients. ${ }^{30}$ All local ethical committees approved the initial trial protocol and gave permission to conduct a 6-year follow-up study. All patients gave written informed consent to the initial study and the follow-up analysis, including the current analysis. The study was also registered in the "Current Controlled Trial Database" (ISRCTN95122877) at http://www.controlled-trials.com and a study protocol was published previously. ${ }^{30}$

\section{Study procedures}

Consecutive adults (age $\geq 18$ years) with a diagnosis of CAP presenting from the community or a nursing home to the ED of one of the participating hospitals were included. All patients fulfilling the following CAP criteria based on the American Thoracic Society guidelines $^{2}$ were eligible: at least one symptom of cough, sputum production, dyspnoea, tachypnoea or pleuritic pain in addition to one finding during auscultation (rales or crepitation) or one infectious sign (core body temperature $>38.0^{\circ} \mathrm{C}$, shivering or white cell count $>10$ or $<4$ cells $\times 10^{9} / \mathrm{L}$ ). The diagnosis of CAP was confirmed in all patients by a new or increasing lung infiltrate on chest X-ray. Inpatients and outpatients were eligible for the study. As previously reported, we included 1381 of 1825 screened patients in the study; of which, 925 had a confirmed diagnosis of CAP and were used for the

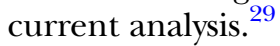

The exclusion criteria were defined as follows: language restriction or dementia precluding informed consent, intravenous drug abuse, severe immunosuppression other than corticosteroids, chronic antibiotic therapy, medical comorbidities with imminent risk of death and hospital acquired pneumonia (defined as newly appearing pulmonary infiltrate $\geq 48$ hours postindex admission or during hospitalisation within 2 weeks before enrolment).

\section{Assessment of vital status and score assignment}

Patients were clinically and biochemically evaluated on admission and throughout the hospital stay. Data on demographics, comorbidities, medication, laboratory variables and imaging as well as vital signs were collected.

Vital status was ascertained by trained medical students by means of phone interviews at days 30,180 and 540 as well as 6 years after discharge. Patients or their household members were contacted first, if not attainable, the general practitioners were called. In cases of missing vital status, patients were categorised as survivors and the latest hospital discharge date derived from medical records was used to calculate the survival time. The decision for ICU transfer was up to the discretion of the treating physicians who were not aware of the NEWS score. We recorded all patients with empyema diagnosed by their treating physicians by ultrasound and laboratory examinations.

For all patients, PSI and CURB-65 scores were calculated on admission to the ED as part of the routine. ${ }^{9} 1021$ The PSI includes 20 variables resulting in a point score and classifies the patients with CAP into five risk classes whereas the CURB-65 score uses a 5-point system (confusion, urea, respiratory rate, blood pressure, age $>65$ years) classifying the patients into three risk classes. NEWS was calculated retrospectively on admission data based on the following six physiological parameters: respiratory rate, oxygen saturation, temperature, systolic blood pressure, pulse rate and level of consciousness. Every continuous variable scores a maximum of 3 points, whereas the need for supplemental oxygen and the level of consciousness are binary coded with zero points if absent/ 
normal and 2 or 3 points if present/altered, respectively. The resulting aggregate divides the patients into three categories with low ( $0-4$ points), medium (5-6 points) or high ( $\geq 7$ points) risk. As an exception, a single physiological parameter scoring 3 points categorises a patient at medium risk instead of low risk, denominated as an RED score.

\section{Statistical analysis}

For the statistical analysis, we used STATA V.12.1 (Stata Corp, College Station, Texas, USA). Statistical significance was defined as a $\mathrm{p}$ value of $<0.05$; two-tailed tests were used.

The categorical variables are presented as percentages (numbers) and the continuous variables are represented

Table 1 Baseline characteristics and outcomes of the study population

\begin{tabular}{|c|c|c|c|c|c|}
\hline \multirow[b]{2}{*}{ Characteristics } & \multirow[b]{2}{*}{$\begin{array}{l}\text { Entire cohort } \\
(n=925)\end{array}$} & \multicolumn{3}{|c|}{ NEWS categories } & \multirow[b]{2}{*}{ p Value } \\
\hline & & Low $(n=349)$ & $\begin{array}{l}\text { Moderate } \\
(n=236)\end{array}$ & High $(n=340)$ & \\
\hline \multicolumn{6}{|l|}{ Demographic characteristics } \\
\hline Age & 73 (59-82) & $67(50-82)$ & 74 (62-83) & 75 (63-82) & $<0.001$ \\
\hline Male & $544(58.8 \%)$ & $195(55.9 \%)$ & $131(55.5 \%)$ & $218(64.1 \%)$ & 0.044 \\
\hline \multicolumn{6}{|l|}{ Comorbidities } \\
\hline Congestive heart failure & $159(17.2 \%)$ & $38(10.9 \%)$ & $44(18.6 \%)$ & $77(22.6 \%)$ & $<0.001$ \\
\hline Chronic renal failure & $206(22.3 \%)$ & $56(16.0 \%)$ & $59(25.0 \%)$ & $91(26.8 \%)$ & 0.002 \\
\hline Diabetes mellitus & $162(17.5 \%)$ & $51(14.6 \%)$ & $45(19.1 \%)$ & $66(19.4 \%)$ & 0.19 \\
\hline COPD & $282(30.5 \%)$ & 75 (21.5\%) & $73(30.9 \%)$ & $134(39.4 \%)$ & $<0.001$ \\
\hline Neoplastic disease & $118(12.8 \%)$ & $42(12.0 \%)$ & $31(13.1 \%)$ & $45(13.2 \%)$ & 0.88 \\
\hline Cerebrovascular disease & $82(8.9 \%)$ & $18(5.2 \%)$ & $23(9.7 \%)$ & $41(12.1 \%)$ & 0.005 \\
\hline Coronary artery disease & $183(19.8 \%)$ & $46(13.2 \%)$ & $53(22.5 \%)$ & $84(24.7 \%)$ & $<0.001$ \\
\hline PAOD & $47(5.1 \%)$ & $13(3.7 \%)$ & $16(6.8 \%)$ & $18(5.3 \%)$ & 0.25 \\
\hline \multicolumn{6}{|l|}{ Clinical history and risk factors } \\
\hline Chills & $301(32.5 \%)$ & $108(35.3 \%)$ & $80(39.6 \%)$ & $113(37.8 \%)$ & 0.71 \\
\hline Fever & $618(67.2 \%)$ & $240(68.8 \%)$ & $152(65.2 \%)$ & $226(67.1 \%)$ & 0.67 \\
\hline Average smoking (pack-years) & $40(20-50)$ & $30(12-50)$ & $35(15-50)$ & $40(30-60)$ & 0.001 \\
\hline \multicolumn{6}{|l|}{ Clinical findings } \\
\hline Confusion & $74(8.8 \%)$ & $0(0.0 \%)$ & $22(10.3 \%)$ & $52(17.0 \%)$ & $<0.001$ \\
\hline Body temperature, ${ }^{\circ} \mathrm{C}$ & $38.1(37.2-38.9)$ & $37.8(37.1-38.6)$ & $37.8(37.1-38.7)$ & $38.5(37.6-39.1)$ & $<0.001$ \\
\hline $\begin{array}{l}\text { Systolic blood pressure, } \\
\mathrm{mm} \mathrm{Hg}\end{array}$ & $132(119-148)$ & $134(120-150)$ & $133(120-148)$ & $130(110-148)$ & 0.001 \\
\hline Peripheral oxygen saturation & $95(92-97)$ & $96.0(94.0-97.0)$ & $96.0(92.5-97.0)$ & $94.0(92.0-96.0)$ & 0.041 \\
\hline Respiratory rate & $20(16-25)$ & $17(15-20)$ & $20(16-24)$ & $25(22-31)$ & $<0.001$ \\
\hline Oxygen therapy, non-invasive & $460(49.7 \%)$ & $81(23.2 \%)$ & $113(47.9 \%)$ & $266(78.2 \%)$ & $<0.001$ \\
\hline \multicolumn{6}{|l|}{ Scores } \\
\hline PSI class I & $104(11.2 \%)$ & $73(20.9 \%)$ & $17(7.2 \%)$ & $14(4.1 \%)$ & $<0.001$ \\
\hline PSI class II & $139(15.0 \%)$ & $74(21.2 \%)$ & $31(13.1 \%)$ & $34(10.0 \%)$ & \\
\hline PSI class III & $180(19.5 \%)$ & $76(21.8 \%)$ & $53(22.5 \%)$ & $51(15.0 \%)$ & \\
\hline PSI class IV & $351(37.9 \%)$ & 97 (27.8\%) & $96(40.7 \%)$ & $158(46.5 \%)$ & \\
\hline PSI class V & $151(16.3 \%)$ & $29(8.3 \%)$ & $39(16.5 \%)$ & $83(24.4 \%)$ & \\
\hline CURB-65 class 0 & $206(22.3 \%)$ & $124(35.5 \%)$ & $45(19.1 \%)$ & 37 (10.9\%) & $<0.001$ \\
\hline CURB-65 class 1 & $253(27.4 \%)$ & $109(31.2 \%)$ & $71(30.1 \%)$ & $73(21.5 \%)$ & \\
\hline CURB-65 class 2 & $306(33.1 \%)$ & $102(29.2 \%)$ & $82(34.7 \%)$ & $122(35.9 \%)$ & \\
\hline CURB-65 class 3 & $134(14.5 \%)$ & $14(4.0 \%)$ & $35(14.8 \%)$ & $85(25.0 \%)$ & \\
\hline CURB-65 class 4 & $25(2.7 \%)$ & $0(0.0 \%)$ & $3(1.3 \%)$ & $22(6.5 \%)$ & \\
\hline CURB-65 class 5 & $1(0.1 \%)$ & $0(0.0 \%)$ & $0(0.0 \%)$ & $1(0.3 \%)$ & \\
\hline \multicolumn{6}{|l|}{ Outcomes } \\
\hline 30-day mortality & $50(5.4 \%)$ & $7(2.0 \%)$ & $16(6.8 \%)$ & $27(7.9 \%)$ & 0.001 \\
\hline 180-day mortality & $106(11.5 \%)$ & $22(6.3 \%)$ & $30(12.7 \%)$ & $54(15.9 \%)$ & $<0.001$ \\
\hline 6-year mortality & $417(45.1 \%)$ & $118(33.8 \%)$ & $115(48.7 \%)$ & $184(54.1 \%)$ & $<0.001$ \\
\hline ICU admission & $83(9.0 \%)$ & $7(2.0 \%)$ & $21(8.9 \%)$ & $55(16.2 \%)$ & $<0.001$ \\
\hline Empyema & 31 (3.4\%) & $5(1.4 \%)$ & $9(3.8 \%)$ & $17(5.0 \%)$ & 0.031 \\
\hline Relapse/re-hospitalisation & $39(4.2 \%)$ & $10(2.9 \%)$ & $13(5.5 \%)$ & $16(4.7 \%)$ & 0.25 \\
\hline Length of stay, days & $8(5-12)$ & $6.0(3.0-10.0)$ & $8.0(6.0-12.0)$ & $10.0(6.0-14.5)$ & $<0.001$ \\
\hline
\end{tabular}

Data are presented as $n(\%)$ or median (IQR). NEWS categories refers to low (0-4 points), medium (5-6 points) or high ( $\geq 7$ points). COPD, chronic obstructive pulmonary disease; CURB- 65 , confusion, urea $>7 \mathrm{mmol} / \mathrm{L}^{-1}$, respiratory frequency $\geq 30 \mathrm{breaths} / \mathrm{min}^{-1}$, low blood pressure (systolic value $<90 \mathrm{~mm} \mathrm{Hg}$ or diastolic value $\leq 60 \mathrm{~mm} \mathrm{Hg}$ ) and age $\geq 65$ years; ICU, intensive care unit; NEWS, National Early Warning Score; PAOD, peripheral artery occlusive disease; PSI, pneumonia severity index. 
as medians (IQRs) with 95\% CIs, wherever applicable. Frequency comparison was estimated by $\chi^{2}$ (Wald) test and two-group comparisons by Mann-Whitney U test.

The primary end point of this study was mortality within 6 years. Mortality was reported at short term (day 30), and long term (day 180 and 6 years). Secondary outcomes were adverse clinical outcomes, including ICU admission, empyema and re-hospitalisation, all occurring within 30 days after randomisation admission.

We used univariate and multivariate regression analyses to assess the association between the prognostic scores and the different outcomes. We report HR for all time-to-event analyses, and ORs for all logistic regression analyses. We calculated different multivariate regression models, including age and gender (model 1) and age, gender and main comorbidities (chronic obstructive pulmonary disease (COPD), congestive heart failure, neoplastic disease, diabetes mellitus, coronary artery disease, cerebrovascular disease, peripheral artery occlusive disease (PAOD), chronic renal failure) (model 2). Discrimination was assessed by means of the area under the receiver operating characteristics (ROC) curve (AUC) with the 95\% CI. For further illustration, we generated Kaplan-Meier plots for mortality and adverse outcomes by NEWS category. For this time-to-event analysis, censoring occurred at the time of death or at the previous contact for patients lost to follow-up.

Finally, we also investigated whether NEWS adds prognostic information to PSI and CURB-65 with regard to discrimination. For this purpose, we compared the AUC of a regression model limited to the PSI score with a binary regression model, including PSI and NEWS. The same was performed for CURB-65.

\section{RESULTS}

\section{Patient population}

Overall, we included 925 CAP patients and the median follow-up was 6.1 years. Baseline characteristics overall and according to NEWS categories (low (0-4 points), medium (5-6 points) or high ( $\geq 7$ points) $)$ risk are presented in table 1 . The study population showed a considerably burden of comorbidities (eg, COPD, chronic renal failure, coronary artery disease), with higher frequency in higher NEWS categories. Most patients were treated as inpatients with $8.8 \%$ of patients being treated on an outpatient basis.

\section{NEWS and mortality outcomes}

The overall 30-day mortality was $5.4 \%$ and increased to $45.1 \%$ after 6 years. The 30-day mortality was significantly higher in NEWS category 3 compared to categories 1 and 2 as presented in table 1 and Kaplan-Meier survival curves (figure 1).

Table 2 shows the unadjusted and adjusted regression analyses assessing the association of NEWS with all-cause mortality at 30 days, 180 days and 6 years. For the 30-day mortality, an increase in NEWS category was associated
30 day survival

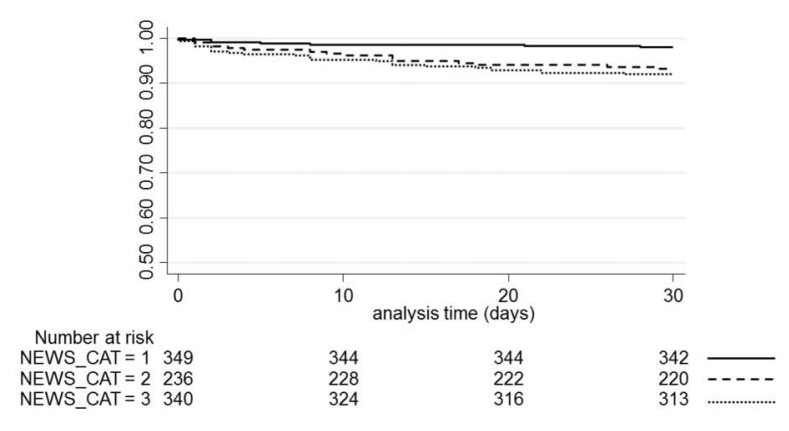

180 day survival

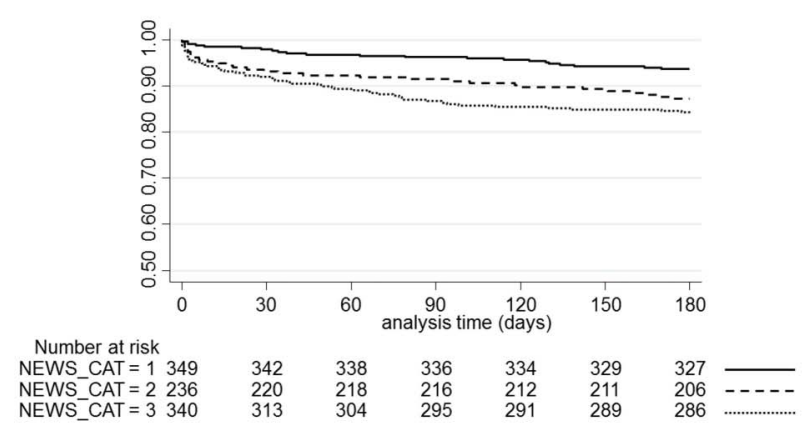

6 year survival

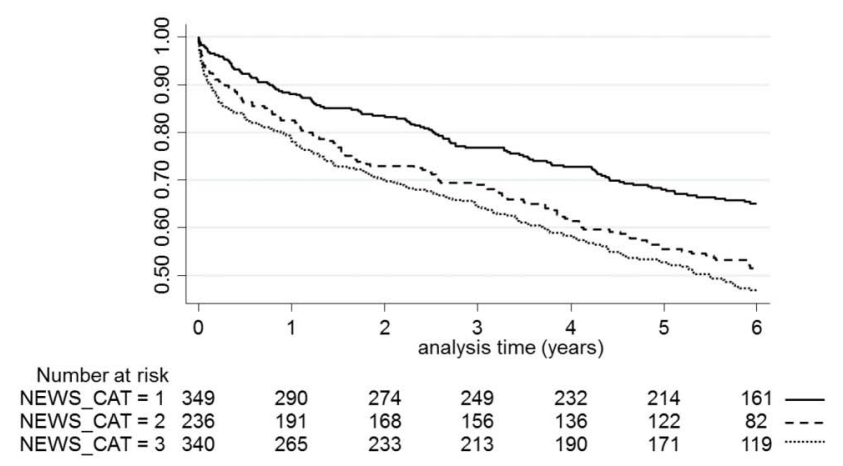

Figure 1 Kaplan-Meier plots showing the association between mortality outcomes and NEWS categories.

with a $16 \%$ increase in odds for reaching the event (OR $1.16,95 \% 1.07$ to $1.27, \mathrm{p}=0.001)$. These results were similar for longer term mortality and also after rigorous adjustment in the different models. Yet, mortality discrimination analysis shows only low results for NEWS with AUCs of $0.65,0.62$ and 0.60 after 30 days, 180 days and 6 years, respectively. In contrast, PSI and CURB-65 showed better mortality discrimination with AUC between 0.76 and 0.80 for PSI and 0.69 and 0.73 for CURB-65. Combining NEWS with PSI or CURB-65 score in a statistical model did not improve the predictive value of these established scores with regard to mortality compared to the scores alone. 
Table 2 NEWS as a mortality predictor compared to the PSI and CURB-65 scores

\begin{tabular}{|c|c|c|c|}
\hline & Mortality 30 days & Mortality 180 days & Mortality 6 years \\
\hline Unadjusted OR & $\begin{array}{l}1.16(1.07 \text { to } 1.27) \\
p=0.001\end{array}$ & $\begin{array}{l}1.13(1.06 \text { to } 1.20) \\
p<0.001\end{array}$ & $\begin{array}{l}1.13(95 \% \mathrm{Cl} 1.08 \text { to } 1.17) \text {, } \\
p<0.001\end{array}$ \\
\hline Adjusted OR (model 1)* & $\begin{array}{l}1.15(1.05 \text { to } 1.25) \\
p=0.003\end{array}$ & $\begin{array}{l}1.11(1.04 \text { to } 1.18) \\
p=0.002\end{array}$ & $\begin{array}{l}1.10(95 \% \mathrm{Cl} 1.05 \text { to } 1.16) \\
\mathrm{p}<0.001\end{array}$ \\
\hline Adjusted OR (model 2)† & $\begin{array}{l}1.10(1.01 \text { to } 1.21) \\
p=0.035\end{array}$ & $\begin{array}{l}1.07(1.00 \text { to } 1.15) \\
p=0.038\end{array}$ & $\begin{array}{l}1.08(95 \% \text { Cl } 1.02 \text { to } 1.13) \text {, } \\
p=0.007\end{array}$ \\
\hline \multicolumn{4}{|l|}{ Discrimination } \\
\hline AUC NEWS & 0.65 (0.58 to 0.72$)$ & $0.62(0.57$ to 0.67$)$ & $0.60(95 \% \mathrm{Cl} 0.57$ to 0.64$)$ \\
\hline AUC PSI & $0.80(0.76$ to 0.84$)$ & $0.76(0.72$ to 0.80$)$ & $0.79(95 \% \mathrm{Cl} 0.76$ to 0.81$)$ \\
\hline NEWS vs PSI & $p<0.001$ & $p<0.001$ & $p<0.001$ \\
\hline AUC NEWS and PSI & $0.82(0.77$ to 0.86$)$ & $0.77(0.73$ to 0.81$)$ & $0.79(95 \% \mathrm{Cl} 0.76$ to 0.82$)$ \\
\hline NEWS and PSI vs PSI & $p=0.084$ & $p=0.074$ & $p=0.911$ \\
\hline AUC CURB-65 & $0.72(0.65$ to 0.78$)$ & $0.69(0.64$ to 0.74$)$ & $0.73(95 \% \mathrm{Cl} 0.69$ to 0.76$)$ \\
\hline NEWS vs CURB-65 & $p=0.076$ & $\mathrm{p}=0.015$ & $p<0.001$ \\
\hline AUC NEWS and CURB-65 & 0.73 (0.67 to 0.79$)$ & $0.70(0.66$ to 0.75$)$ & $0.73(95 \% \mathrm{Cl} 0.70$ to 0.76$)$ \\
\hline $\begin{array}{l}\text { NEWS and CURB-65 vs } \\
\text { CURB-65 }\end{array}$ & $p=0.178$ & $\mathrm{p}=0.091$ & $p=0.29$ \\
\hline \multicolumn{4}{|c|}{$\begin{array}{l}\text { Data from univariate and multivariate analysis are given as } \mathrm{OR}(95 \% \mathrm{Cl}) \text { per point increase, } \mathrm{p} \text { value. Data from the ROC analysis are given as } \\
\text { AUC }(95 \% \mathrm{Cl}) \text { or } \mathrm{p} \text { value. } \\
\text { *Adjusted for age, gender. } \\
\text { †Adjusted for age, gender, comorbidities (COPD, congestive heart failure, neoplastic disease, diabetes mellitus, coronary artery disease, } \\
\text { cerebrovascular disease, PAOD, chronic renal failure). } \\
\text { AUC, area under the curve; CURB- } 65 \text { : confusion, urea }>7 \mathrm{mmol} / \mathrm{L}^{-1} \text {, respiratory frequency } \geq 30 \text { breaths } / \mathrm{min}^{-1} \text {, low blood pressure (systolic } \\
\text { value }<90 \mathrm{~mm} \mathrm{Hg} \text { or diastolic value } \leq 60 \mathrm{~mm} \mathrm{Hg} \text { ) and age } \geq 65 \text { years; NEWS, National Early Warning Score; PSI, pneumonia severity index. }\end{array}$} \\
\hline
\end{tabular}

\section{NEWS and adverse clinical outcomes}

The risk for ICU admission and empyema significantly increased with increasing NEWS categories. Figure 2 shows a significant separation in time to ICU admission with increasing NEWS categories.

Table 3 shows the unadjusted and adjusted regression analysis investigating the association of NEWS with adverse clinical outcomes, namely ICU admission, empyema and re-hospitalisation. The results were statistically significant for NEWS as a predictor for ICU admission (OR 1.29 (1.20 to 1.39)) and empyema (OR 1.16 (1.04 to 1.29)) within 30 days after admission. This was also true after adjustment for age, gender and comorbidities $(\mathrm{p}<0.01$, each). Concerning re-hospitalisation, no significant association was found.

In regard to discrimination, NEWS showed the highest AUC for all three outcomes compared to PSI and CURB-65. For ICU admission, NEWS significantly improved PSI (from AUC 0.66 to $0.74, \mathrm{p}=0.001$ ) and CURB-65 (from AUC 0.64 to 0.73, $\mathrm{p}=0.002$ ). For empyema, NEWS also tended to improve PSI (from AUC 0.50 to $0.64, \mathrm{p}=0.086)$ and significantly improved CURB-65 (from AUC 0.50 to 0.65, $\mathrm{p}=0.025$ ). For re-hospitalisation, no significant improvement was found.

Patients who were misclassified by the PSI score as low risk (PSI class 1 or 2) but correctly identified by NEWS had younger age (median age 49 vs 74 years), less comorbidities (heart and renal failure, coronary heart disease) and more frequent deterioration (chills, oxygenation) of vital signs compared to patients who were correctly identified by both scores.

\section{DISCUSSION}

This first study evaluating NEWS in a large population with CAP from a multicentre study with a 6-year follow-up has three key findings. First, NEWS is a moderate predictor for adverse clinical outcomes, particularly ICU admission, and to a lesser degree for empyema in patients presenting with CAP to the ED. Second, NEWS improves the PSI and CURB-65 for prediction of ICU admission. Third, although NEWS is associated with mortality, this score has a lower prognostic performance compared to standard CAP scores and did not improve their performance.

NEWS has been originally established and validated as a track-and-trigger system for acute illness. A first study showed its superiority comparing it to other EWS. ${ }^{21}{ }^{22}$ Most subsequent research validated the superior performance of NEWS compared to other algorithms. ${ }^{23-25} 27$ Also the different parameters included in NEWS were well validated. ${ }^{31-33}$ Yet, the performance of NEWS within specific patient subpopulations has not well been studied, with some exceptions such as patients with severe sepsis or septic shock. ${ }^{28}$ Most validation studies were singlecentre studies with a short follow-up of patients. Thus, external validity and long-term predictive ability of NEWS remains unknown today.

Reflecting on the data of our clinical findings (see table 1), mortality and adverse clinical outcomes occurred more frequently in higher NEWS categories, confirming the basic utility of NEWS as a severity indicator. However, a majority of the clinical trials were performed in a heterogeneous patient population with 
ICU-admission 30 days after admission

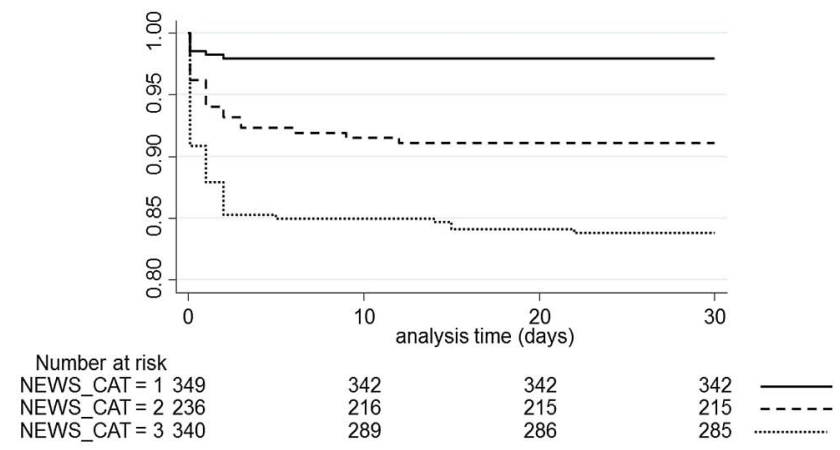

Complications 30 days after admission

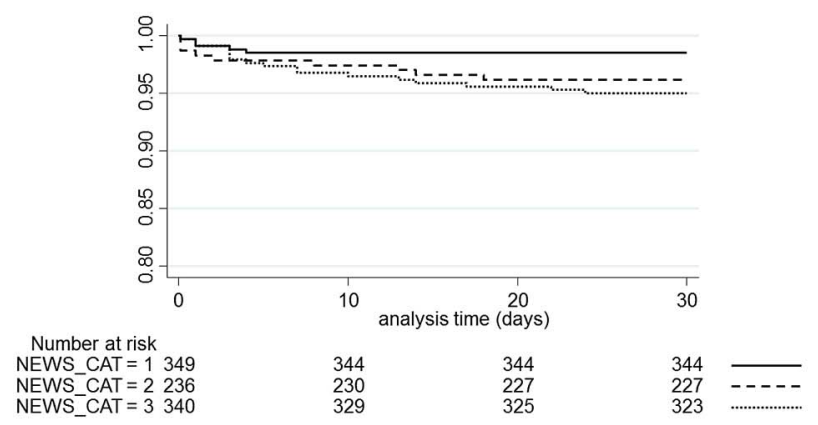

Re-Hospitalisation 30 days after admission

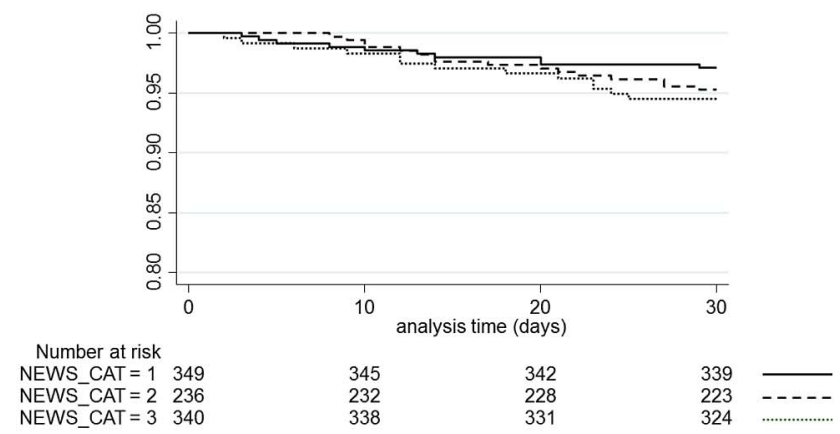

Figure 2 Kaplan-Meier plots showing the association between adverse outcomes and NEWS categories.

diverse principal morbidities. ${ }^{22} \quad 25-28 \quad 31-33$ Our study focused on patients with CAP, a disease with a relatively high short-term mortality. ${ }^{44}$ Therefore, early recognition of severity is crucial for further patient management and the use of predictive tools is currently recommended by American and European guidelines. ${ }^{2}{ }^{13}$ Our analyses reveal a moderate predictive value for a 30-day ICU admission and empyema using NEWS. Despite the rather aged patient population with a high burden of comorbidities, results remained significant after adjustment for these factors. This main finding supports the routine use of NEWS in CAP patients. Interestingly, the PSI contains similar physiological parameters as used for NEWS calculation. Still, NEWS was superior for adverse outcome prediction but inferior with regard to mortality prediction. This may be explained by the fact that PSI is age dominated and while age is a good predictor for mortality, aged people at the end of life may be less often admitted to the ICU. NEWS sets the main focus on the acute condition (eg, need for supplemental oxygen or altered level of consciousness) allowing better evaluation for the eventual need for ICU admission. Interestingly, in line with this, we found that younger patients with lower burden of comorbidities and more severe deterioration of vital signs were at higher risk for being misclassified as 'low risk' with PSI but correctly identified with NEWS. This patient population may thus show the most benefit of combination of both scores.

Furthermore, we found that combining NEWS with established CAP-specific scores in a joint regression model improves the prognostic accuracy regarding 30-day ICU admission. The application of the PSI in patients with CAP is widespread in the USA, whereas the CURB-65 is mostly used in Europe. Our data support the calculation of both scores on admission to the ED in the CAP patient population. Although, this may increase resource use, EWS and CAP scores are routinely calculated in many hospitals. Indeed, further studies should be conducted to compare patient management based on these combined scores to routine care to ultimately understand the benefit for patients.

Most of the previous studies analysed and proved association between NEWS and short-term mortality at maximal 30 days. ${ }^{22} 2325$ In our regression models for mortality outcomes, we could show an association of NEWS with 30-day, 180-day and 6-year mortality. However, PSI and CURB-65 were superior as mortality predictors. Probably this is due to the simple six-point system of basic physiological parameter reflecting the very acute condition of a patient and thus the trigger-and-track nature of the NEWS. Whereas the PSI and CURB-65 scores include more variables taking into consideration the all-over morbidity of the patient (eg, age, comorbidities, laboratory parameters), giving them an advantage about mortality prediction beyond the emergency setting.

The strength of our study is the considerable patient numbers originating from a multicentre setting with well-defined CAP criteria and a consistent distribution to the three NEWS categories. Furthermore, the long followup of 6 years with repeated telephone interviews allows the investigation of short- and long-term outcomes, while most previous studies focused on short-term data. There are, however, several limitations to this report. Despite the multicentre character, the study was conducted exclusively in Switzerland with predominantly Caucasian patients limiting generalisability. Furthermore, this was a secondary analysis of a previous trial which had some exclusion 
Table 3 NEWS as adverse outcome predictor compared to the PSI and CURB-65 scores

$\begin{array}{lll}\begin{array}{l}\text { ICU admission } \\ \text { Within } \mathbf{3 0} \text { days }\end{array} & \begin{array}{l}\text { Empyema } \\ \text { Within } \mathbf{3 0} \text { days }\end{array} & \begin{array}{l}\text { Re-hospitalisation } \\ \text { Within } \mathbf{3 0} \text { days }\end{array}\end{array}$

\begin{tabular}{llll}
\hline Unadjusted OR & $1.29(1.20$ to 1.39$), p<0.001$ & $1.16(1.04$ to 1.29$), p=0.007$ & 1.08 ( 0.98 to 1.18$), p=0.143$ \\
Adjusted OR (model 1$)^{*}$ & $1.30(1.20$ to 1.40$), p<0.001$ & $1.18(1.06$ to 1.32$), p=0.003$ & 1.08 (0.98 to 1.20$), p=0.106$ \\
Adjusted OR (model 2) $\dagger$ & $1.27(1.18$ to 1.37$), p<0.001$ & $1.17(1.05$ to 1.30$), p=0.005$ & 1.07 (0.97 to 1.18$), p=0.184$ \\
Discrimination & & & \\
AUC NEWS & $0.73(0.67$ to 0.78$)$ & $0.64(0.54$ to 0.73$)$ & $0.58(0.49$ to 0.66$)$ \\
AUC PSI & $0.66(0.60$ to 0.72$)$ & $0.50(0.40$ to 0.60$)$ & $0.53(0.43$ to 0.63$)$ \\
NEWS vs PSI & $\mathrm{p}=0.072$ & $\mathrm{p}=0.042$ & $\mathrm{p}=0.358$ \\
AUC NEWS and PSI & $0.74(0.69$ to 0.79$)$ & $0.64(0.54$ to 0.73$)$ & $0.58(0.49$ to 0.66$)$ \\
NEWS and PSI vs PSI & $\mathrm{p}=0.001$ & $\mathrm{p}=0.086$ & $\mathrm{p}=0.414$ \\
AUC CURB-65 & $0.64(0.58$ to 0.70$)$ & $0.50(0.40$ to 0.59$)$ & $0.50(0.41$ to 0.59$)$ \\
NEWS vs CURB-65 & $\mathrm{p}=0.015$ & $\mathrm{p}=0.011$ & $\mathrm{p}=0.118$ \\
AUC NEWS and CURB-65 & $0.73(0.68$ to 0.79$)$ & $0.65(0.55$ to 0.74$)$ & $0.58(0.49$ to 0.67$)$ \\
NEWS and CURB-65 vs CURB-65 & $\mathrm{p}=0.002$ & $\mathrm{p}=0.025$ & $\mathrm{p}=0.246$ \\
\hline
\end{tabular}

Data from univariate and multivariate analysis are given as OR $(95 \% \mathrm{Cl})$ per point increase. Data from the ROC analysis are given as AUC $(95 \% \mathrm{Cl})$ or $\mathrm{p}$ value.

*Adjusted for age, gender.

†Adjusted for age, gender, comorbidities (COPD, congestive heart failure, neoplastic disease, diabetes mellitus, coronary artery disease, cerebrovascular disease, PAOD, chronic renal failure).

AUC, area under the curve; CURB-65, confusion, urea $>7 \mathrm{mmol} / \mathrm{L}^{-1}$, respiratory frequency $\geq 30$ breaths $/ \mathrm{min}^{-1}$, low blood pressure (systolic value $<90 \mathrm{~mm} \mathrm{Hg}$ or diastolic value $\leq 60 \mathrm{~mm} \mathrm{Hg}$ ) and age $\geq 65$ years; ICU, intensive care unit; NEWS, National Early Warning Score; PSI, pneumonia severity index.

criteria inducing potential confounding. NEWS has been primarily recommended to be used as a trigger score for patient deterioration during hospital stay and not in the initial setting. ${ }^{21}$ Since parameters for calculation of NEWS were collected only upon admission to the ED, no follow-up analyses were carried out.

\section{CONCLUSION}

We found NEWS to provide additional prognostic information with regard to risk of ICU admission and empyema, thereby improving traditional clinical CAP risk scores in the management of patients in the ED setting.

\author{
Author affiliations \\ ${ }^{1}$ University Department of Medicine, Kantonsspital Aarau, Aarau, Switzerland \\ ${ }^{2}$ Department of Internal Medicine, Division of Endocrinology, Diabetes and \\ Clinical Nutrition, University Hospital Basel, Basel, Switzerland \\ ${ }^{3}$ Department of Internal Medicine, Bürgerspital Solothurn, Solothurn, \\ Switzerland \\ ${ }^{4}$ Basel University Medical Clinic Liestal, Liestal, Switzerland \\ ${ }^{5}$ Department of Internal Medicine, Kantonsspital Münsterlingen, \\ Münsterlingen, Switzerland \\ ${ }^{6}$ Department of Internal Medicine, Kantonsspital Lucerne, Lucerne, \\ Switzerland
}

Acknowledgements The authors are thankful to the ED, medical clinic and central laboratory staff of the University Hospital Basel and the Cantonal Hospitals Aarau, Liestal, Lucerne and Muensterlingen, and the 'Buergerspital' Solothurn for their assistance and technical support. In particular, they thank all patients, patients' relatives and all local general practitioners who participated in this study. Finally, they acknowledge the ProHOSP Study Group for their important support.

Collaborators The ProHOSP Study Group members: U. Schild, K. Regez, R. Bossart, C. Blum, M. Wolbers, S. Neidert, I. Suter, H.C. Bucher, F. Mueller, A. Chaudry, J. Haeuptle, R. Zarbosky, R. Fiumefreddo, M. Wieland,
C. Nussbaumer, A. Christ, R. Bingisser, and K. Schneider (University Hospital Basel, Basel, Switzerland); T. Bregenzer, D. Conen, A. Huber, and J. Staehelin (Kantonsspital Aarau, Aarau, Switzerland); W. Zimmerli,

C. Falconnier, and C. Bruehlhardt (Kantonsspital Liestal, Liestal, Switzerland); C. Henzen and V. Briner (Kantonsspital Luzern, Luzern, Switzerland); T. Fricker, C. Hoess, M. Krause, I. Lambinon, and M. Zueger (Kantonsspital Muensterlingen, Muensterlingen, Switzerland); and R. Thomann, R. Schoenenberger, and R. Luginbuehl (Buergerspital Solothurn, Solothurn, Switzerland).

Contributors All authors made substantive intellectual contributions to this study. DS-R, AK, PS and BM had the idea for and conducted statistical analyses and drafted the first manuscript. MC-C, RT, WZ, CHo and CHe were in charge of acquisition of patient data during the trial and provided individual patient data from their hospitals. For this manuscript, they have made substantial contributions to conception and design, and have taken an active part in acquisition, analysis and interpretation of data. All authors contributed to the interpretation of data and to the revising of the manuscript critically for important intellectual content. All authors approved the final version of the manuscript, and agreed to be accountable for all aspects of the work in ensuring that questions related to the accuracy or integrity of any part of the work are appropriately investigated and resolved.

Funding The initial study was mainly funded by the Swiss National Science Foundation (grant number SNF 3200B0-116177/1), Santé Suisse and the Gottfried and Julia Bangerter-Rhyner Foundation.

Competing interests $\mathrm{AK}, \mathrm{MC}-\mathrm{C}, \mathrm{BM}$ and PS received support from B.R.A.H.M.S/Thermo Scientific Biomarkers and BioMerieux to attend meetings and fulfil speaking engagements. PS and BM received unrestricted research grants from B.R.A.H.M.S/Thermo Scientific Biomarkers and BioMerieux, and BM has served as a consultant to B.R.A.H.M.S/Thermo Scientific Biomarkers and BioMerieux. All other authors have no relationships to industry relevant to this paper.

Ethics approval EKBB, Ethikkommission beider Basel; Kantonale Ethikkommission Aargau/Solothurn; Ethikkommission des Kantons Luzern; Ethikkommission des Kantons Thurgau.

Provenance and peer review Not commissioned; externally peer reviewed. Data sharing statement No additional data are available. 
Open Access This is an Open Access article distributed in accordance with the Creative Commons Attribution Non Commercial (CC BY-NC 4.0) license, which permits others to distribute, remix, adapt, build upon this work noncommercially, and license their derivative works on different terms, provided the original work is properly cited and the use is non-commercial. See: http:// creativecommons.org/licenses/by-nc/4.0/

\section{REFERENCES}

1. Wasson $\mathrm{JH}$, Sox HC, Neff RK, et al. Clinical prediction rules Applications and methodological standards. N Engl J Med 1985;313:793-9.

2. Niederman MS, Mandell LA, Anzueto A, et al. Guidelines for the management of adults with community-acquired pneumonia. Diagnosis, assessment of severity, antimicrobial therapy, and prevention. Am J Respir Crit Care Med 2001;163:1730-54.

3. Musher DM, Thorner AR. Community-acquired pneumonia. N Engl J Med 2014;371:1619-28.

4. Almirall J, Bolibar I, Vidal J, et al. Epidemiology of community-acquired pneumonia in adults: a population-based study. Eur Respir J 2000;15:757-63.

5. Ortqvist A, Hedlund J, Grillner L, et al. Aetiology, outcome and prognostic factors in community-acquired pneumonia requiring hospitalization. Eur Respir J 1990;3:1105-13.

6. Fine MJ, Hough LJ, Medsger AR, et al. The hospital admission decision for patients with community-acquired pneumonia. Results from the pneumonia Patient Outcomes Research Team cohort study. Arch Intern Med 1997;157:36-44.

7. Labarere J, Stone RA, Obrosky DS, et al. Comparison of outcomes for low-risk outpatients and inpatients with pneumonia: a propensity-adjusted analysis. Chest 2007;131:480-8.

8. Daley J, Jencks S, Draper D, et al. Predicting hospital-associated mortality for Medicare patients. A method for patients with stroke, pneumonia, acute myocardial infarction, and congestive heart failure. JAMA 1988;260:3617-24.

9. Fine MJ, Auble TE, Yealy DM, et al. A prediction rule to identify low-risk patients with community-acquired pneumonia. $N$ Engl J Med 1997;336:243-50.

10. Lim WS, van der Eerden MM, Laing $R$, et al. Defining community acquired pneumonia severity on presentation to hospital: an international derivation and validation study. Thorax 2003;58:377-82.

11. Espana PP, Capelastegui A, Gorordo I, et al. Development and validation of a clinical prediction rule for severe community-acquired pneumonia. Am J Respir Crit Care Med 2006;174:1249-56.

12. Liapikou A, Ferrer M, Polverino E, et al. Severe community-acquired pneumonia: validation of the Infectious Diseases Society of America/ American Thoracic Society guidelines to predict an intensive care unit admission. Clin Infect Dis 2009;48:377-85.

13. Woodhead M, Blasi F, Ewig S, et al. Guidelines for the management of adult lower respiratory tract infections. Eur Respir $J$ 2005:26:1138-80.

14. Atlas SJ, Benzer TI, Borowsky LH, et al. Safely increasing the proportion of patients with community-acquired pneumonia treated as outpatients: an interventional trial. Arch Intern Med 1998;158:1350-6.

15. Marrie TJ, Lau CY, Wheeler SL, et al. A controlled trial of a critical pathway for treatment of community-acquired pneumonia. CAPITAL Study Investigators. Community-Acquired Pneumonia Intervention Trial Assessing Levofloxacin. JAMA 2000;283:749-55.

16. Carratala J, Fernandez-Sabe N, Ortega L, et al. Outpatient care compared with hospitalization for community-acquired pneumonia: a randomized trial in low-risk patients. Ann Intern Med 2005;142:165-72.
17. Yealy DM, Auble TE, Stone RA, et al. Effect of increasing the intensity of implementing pneumonia guidelines: a randomized, controlled trial. Ann Intern Med 2005;143:881-94.

18. Labarere J, Stone RA, Scott Obrosky D, et al. Factors associated with the hospitalization of low-risk patients with community-acquired pneumonia in a cluster-randomized trial. J Gen Intern Med 2006;21:745-52.

19. Renaud B, Coma E, Labarere J, et al. Routine use of the Pneumonia Severity Index for guiding the site-of-treatment decision of patients with pneumonia in the emergency department: a multicenter, prospective, observational, controlled cohort study. Clin Infect Dis 2007;44:41-9.

20. Yandiola PP, Capelastegui A, Quintana J, et al. Prospective comparison of severity scores for predicting clinically relevan outcomes for patients hospitalized with community-acquired pneumonia. Chest 2009;135:1572-9.

21. Royal College of Physicians London. National Early Warning Score (NEWS): Standardising the assessment of acute-illness severity in the NHS. Report of a working party. Royal College of Physicians, 2012.

22. Smith GB, Prytherch DR, Meredith $\mathrm{P}$, et al. The ability of the National Early Warning Score (NEWS) to discriminate patients at risk of early cardiac arrest, unanticipated intensive care unit admission, and death. Resuscitation 2013;84:465-70.

23. Badriyah T, Briggs JS, Meredith $P$, et al. Decision-tree early warning score (DTEWS) validates the design of the National Early Warning Score (NEWS). Resuscitation 2014;85:418-23.

24. Tirkkonen J, Olkkola KT, Huhtala $\mathrm{H}$, et al. Medical emergency team activation: performance of conventional dichotomised criteria versus national early warning score. Acta Anaesthesiol Scand 2014:58:411-19.

25. Abbott TE, Vaid N, Ip D, et al. A single-centre observational cohort study of admission National Early Warning Score (NEWS). Resuscitation 2015:92:89-93.

26. Silcock DJ, Corfield AR, Gowens PA, et al. Validation of the Nationa Early Warning Score in the prehospital setting. Resuscitation 2015;89:31-5.

27. Alam N, Vegting IL, Houben E, et al. Exploring the performance of the national early warning Score (NEWS) in a European emergency department. Resuscitation 2015;90:111-15.

28. Keep JW, Messmer AS, Sladden R, et al. National early warning score at Emergency Department triage may allow earlier identification of patients with severe sepsis and septic shock: a retrospective observational study. Emerg Med J 2016;33:37-41.

29. Schuetz P, Christ-Crain M, Thomann R, et al. Effect of procalcitonin-based guidelines vs standard guidelines on antibiotic use in lower respiratory tract infections: the ProHOSP randomized-controlled trial. JAMA 2009;302:1059-66.

30. Schuetz $\mathrm{P}$, Christ-Crain M, Wolbers $\mathrm{M}$, et al. Procalcitonin guided antibiotic therapy and hospitalization in patients with lower respiratory tract infections: a prospective, multicenter, randomized-controlled trial. BMC Health Serv Res 2007;7:102.

31. Eccles SR, Subbe C, Hancock D, et al. CREWS: improving specificity whilst maintaining sensitivity of the National Early Warning Score in patients with chronic hypoxaemia. Resuscitation 2014;85:109-11.

32. Jarvis S, Kovacs C, Briggs J, et al. Aggregate National Early Warning Score (NEWS) values are more important than high scores for a single vital signs parameter for discriminating the risk of adverse outcomes. Resuscitation 2015;87:75-80.

33. Kolic I, Crane S, McCartney S, et al. Factors affecting response to national early warning score (NEWS). Resuscitation 2015;90:85-90.

34. Menendez R, Torres A, Zalacain R, et al. Risk factors of treatment failure in community acquired pneumonia: implications for disease outcome. Thorax 2004;59:960-5. 